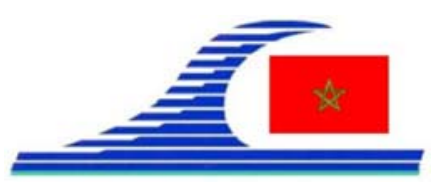

\author{
Conférence Méditerranéenne Côtière et Maritime \\ EDITION 2, TANGER, MAROC (2011) \\ Coastal and Maritime Mediterranean Conference \\ Disponible en ligne - http://www.paralia.fr - Available online
}

\title{
Qualité des eaux d'un lac dans une région côtière de l'est algérien : cas du lac Fetzara
}

\author{
Sameh HABES ${ }^{1}$, Larbi DJABRI ${ }^{2}$, Yacine DJABRI ${ }^{3}$
}

1. Université de Ouargla, Département de Géologie, Algérie.

habes_sameh@yahoo.fr

2. Université Badji Mokhtar, Laboratoire Sécurité Environnementale et Alimentaire.

Larbi_djabri@yahoo.fr

3. CHU, Ibn Rochd, Annaba, Service Gynécologie Obstétrique.

\section{Résumé :}

La lac Fetzara (site RAMSAR du nord de l'Algérie) a des eaux de salinité très variable. Celle-ci est générée par plusieurs facteurs tel que : (i) la géologie, car les différentes formations à l'affleurement influencent différemment la composition chimique de l'eau, (ii) les facteurs climatiques, et (iii) la proximité de la mer par le biais des embruns marins.

\section{Mots clés :}

Lac Fetzara - Salinité - Embruns marins - Evaporation - Eléments majeurs - Eléments en traces

\section{Introduction}

Le lac Fetzara classé site RAMSAR, abrite pendant la saison hivernale des oiseaux migrateurs. Ce label RAMSAR requiert une préservation de cet écosystème aquatique, car il constitue un enjeu international. Afin d'assurer une protection de ce site, un contrôle permanent de la qualité des eaux du lac s’impose. Le présent travail porte sur la qualité des eaux transitant par le lac (entrées et sorties), ce qui nous permet de comprendre leurs évolutions spatio-temporelles.

Situation géographique et Géologique: Le lac Fetzara, est une vaste dépression (figure 1), bordée au nord par le massif de l'Edough, au sud par les monts d'Ain Berda, vers l'ouest, par un faible ressaut de quelques mètres seulement plus élevés que le reste de la plaine et en allant vers l'est on trouve la plaine d'Annaba. Le lac est une dépression nettement dissymétrique, due à une tectonique active pendant le Quaternaire, il fait 14000 ha (15 km de long sur 9 km de large) de superficie. Actuellement, un canal d'assèchement long de14 km traverse le lac dans le sens W-E et rejoint le cours moyen de la Meboudja assurant la vidange du lac.

Le nord-ouest de la région est occupé par les formations métamorphiques, particulièrement riches en gneiss, schistes, micaschistes et cipolins. Ailleurs affleurent les formations sédimentaires constituées d'alluvions sableuses. 
La connaissance de la Mer :

un vecteur du développement durable en Méditerranée

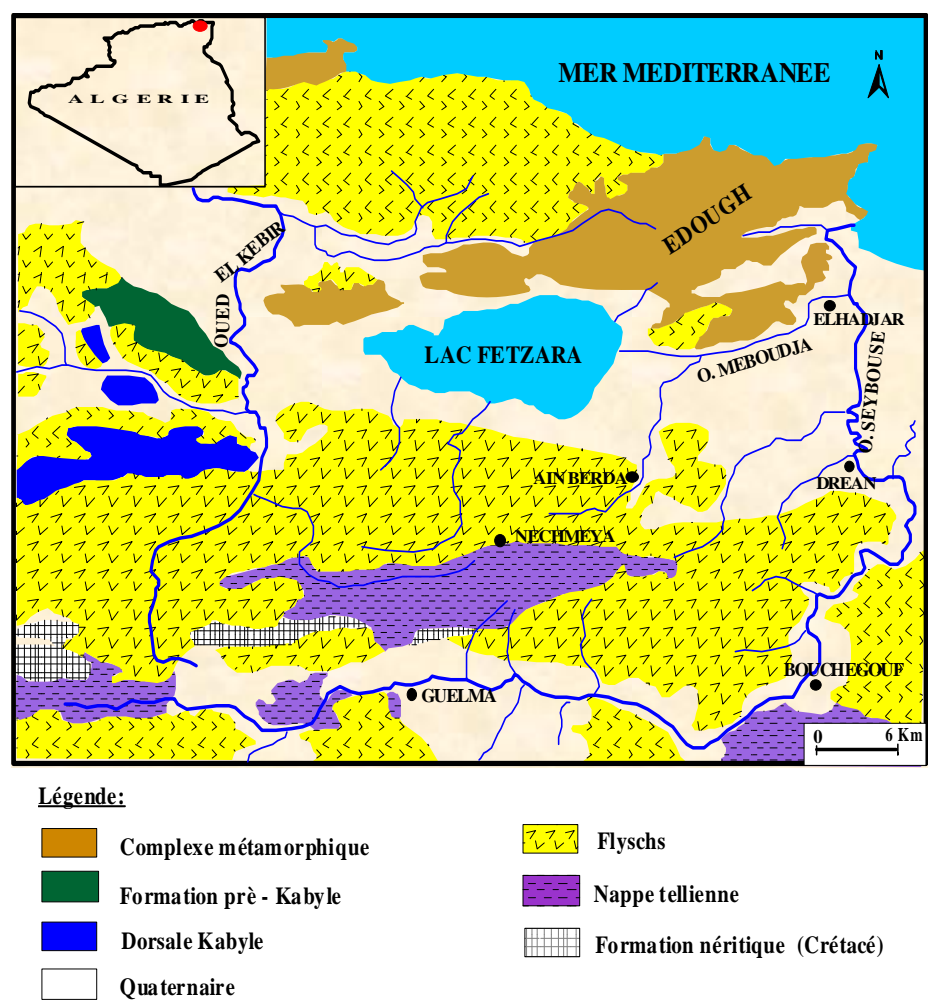

Figure 1. Situation géographique et géologie de la région du Lac Fetzara.

Carte des infiltrations : l'observation de la carte (figure 2), montre que les valeurs de l'infiltration varient entre $4 \mathrm{~mm}$ et plus de $30 \mathrm{~mm}$ et diffèrent d'une zone à l'autre. Elle est de l'ordre de $9 \mathrm{~mm}$ au sud-est et atteint $30 \mathrm{~mm}$ au nord. Cette variation de l'infiltration est favorisée par la perméabilité des sols et la sollicitation de la nappe, cette variation va conditionner les écoulements, la fluctuation des nappes et le déplacement du polluant.

Piézométrie : la carte piézométrique (figure 3), montre un écoulement dirigé vers le lac Fetzara dans sa partie centrale, par contre au niveau de la zone est l'écoulement est dirigé vers l’Oued Meboudja.

\section{Résultats et discussions}

Pour mettre en évidence les liens existant entre les éléments chimiques, nous avons réalisé une analyse en composantes principales.

Observation du cercle ACP (figure 4) : Le cercle réalisé par les deux axes F1F2 (66\%), montre selon l'axe horizontal F1 (48\% de la variance), une opposition entre les eaux fortement minéralisées (riches en chlorures, sulfates, sodium, calcium, magnésium et à degré moindre en bicarbonates), occupant la partie négative de l'axe F1. Selon l'axe F2 (18\% de la variance) on observe une opposition entre les alcalins et les alcalino-terreux. Les premiers se caractérisent par un faciès chloruré sodique et les seconds donnent le faciès sulfaté calcique ou magnésien, s’expliquant par une origine géologique. 


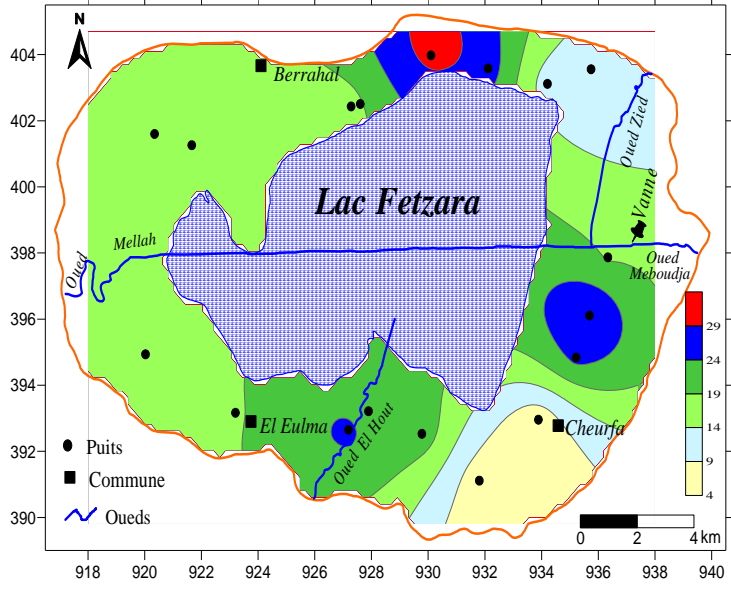

Figures 2. Carte de l'infiltration efficace de la région du Fetzara.

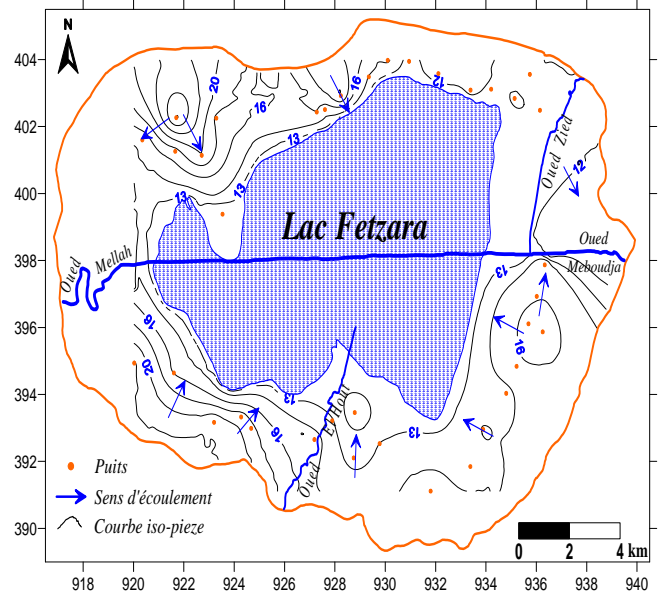

Figures 3. Carte de la piézométrie de la région du Fetzara.

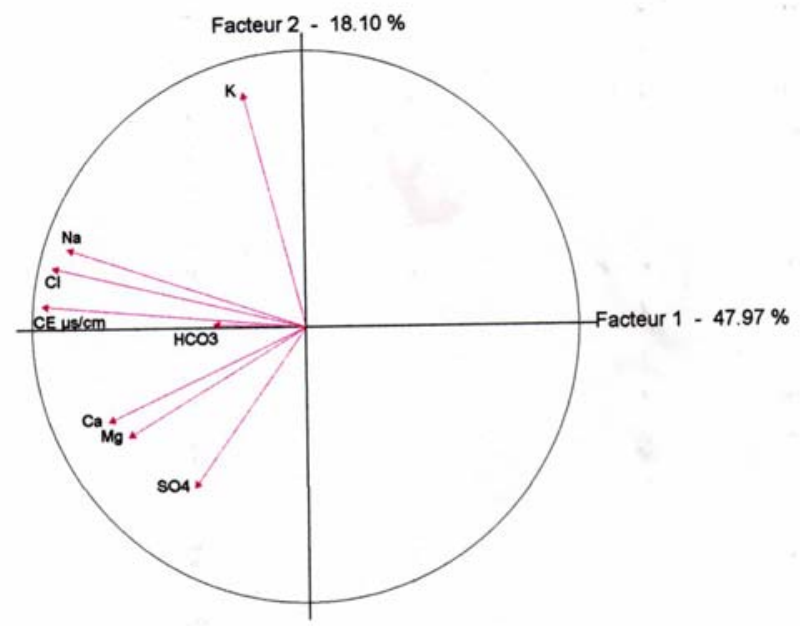

Figure 4. Cercle ACP, Lac Fetzara.

Le lac situé à 15 kilomètres de la mer, est soumis aux effets du climat, caractérisé par une forte évaporation. Par ailleurs les eaux aboutissant au lac, traversent diverses formations géologiques, générant ainsi la salinité observée. Nous allons nous intéresser à l'influence des ces facteurs.

Apports du Strontium: le strontium, indique une salinité d'origine géologique provoquée par les formations évaporitiques. Appliqué aux eaux du lac, on remarque que le rapport $\mathrm{Sr}^{2+} / \mathrm{Ca}^{2}$, oscille entre 1 et 5\%o, montrant une influence localisée des formations évaporitiques, particulièrement au niveau de la zone sud-est du lac (HABES, 2006)

L'influence Marine: Les chlorures sont largement répandus dans la nature, généralement sous forme de sels de sodium $(\mathrm{NaCl})$ et de potassium $(\mathrm{KCl})$. L'ion chlorure est la forme stable dominante des isotopes du chlore, qui n'est pratiquement jamais impliquée dans les processus redox naturels. C’est pourquoi, on s’est intéressé à 
La connaissance de la Mer :

un vecteur du développement durable en Méditerranée

cet élément. Pour mettre en évidence l’influence de la mer sur la qualité des eaux, nous avons étudié la répartition de certains éléments par rapport à la droite des chlorures, eau des précipitations et eau de mer.

L'observation du graphe A et B, (figure 5) montre un alignement des points analysés au-dessus de la droite de référence, ceci nous permet de dire que les éléments peuvent avoir une origine probablement marine. Au niveau du graphe $\mathrm{C}$, les points analysés, se répartissent de part et d'autre de la droite, ce qui met en évidence deux origines du calcium, la première marine et la seconde géologique. Cette répartition, met en évidence une contamination marine, elle serait générée par les embruns marins. Le lac étant situé à environ 15 kilomètres du littoral, il est souvent couvert par des brouillards facilitant la précipitation des sels contenus dans les eaux de mer. Par ailleurs au niveau du lac l'évaporation intense, accroit la salinité des eaux, confirmée par l'étude isotopique réalisée par DJABRI (1996) et par DJABRI et al. (1996).

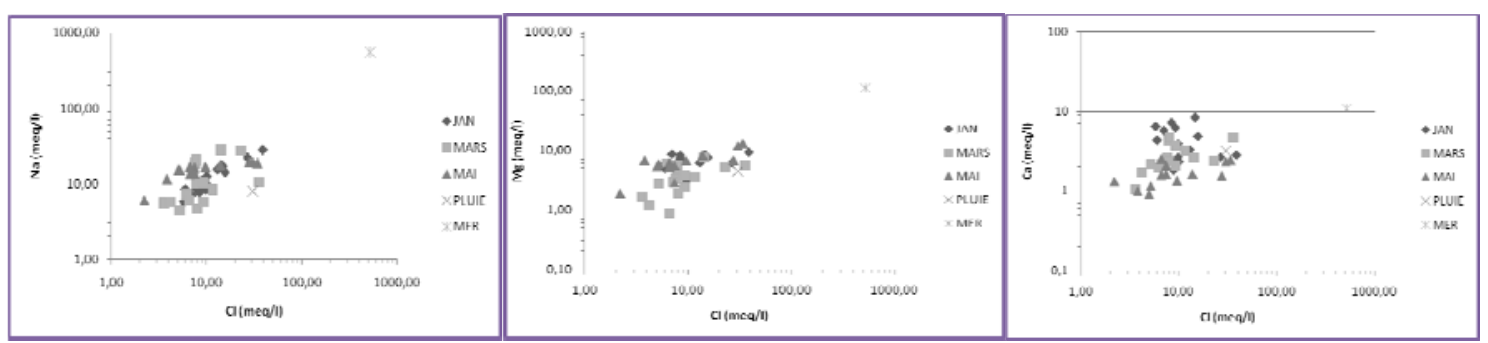

(A)

(B)

(C)

Figure 5. Variations des éléments par rapport à la droite Cl mer-Cl précipitations.

\section{Conclusion}

L'étude réalisée sur les eaux du lac, a montré une compétition entre les alcalins et les alcalino-terreux par l'ACP. Par ailleurs la combinaison de plusieurs paramètres (rapport $\mathrm{Sr}^{2+} / \mathrm{Ca}^{2+}$, la droite $\mathrm{Cl}$ eau de pluie, $\mathrm{Cl}$ eau de mer, ...), indique une origine naturelle de la salinité. Du fait que les eaux sont superficielles, ceci implique une vulnérabilité des eaux aux variations externes, tel que l'évaporation et le brouillard.

\section{Références bibliographiques}

DJABRI L. (1996). Mécanismes de la pollution et vulnérabilité des eaux de la Seybouse - Origines géologiques, industrielles, agricoles et urbaines. Doc Es Sci. Univ. Annaba, $280 \mathrm{p}$.

DJABRI L., MANIA J., MESSADI D., HANI A., SOUAG M. (1996). Apport des isotopes pour la connaissance de l'origine des eaux de la vallée de la Seybouse (est.Algérien). Revista Hidrogeología (Espagne), 12, pp 3-14

HABES S. (2006). Pollution saline d'un lac, cas du lac Fedzara "Est algérien". Mémoire de Magister, Université de Annaba, 105 p. 